Bull. Austral. Math. Soc.

VOL. 38 (1988) [221-229]

\title{
FUBINI'S THEOREM FOR RADON POLYMEASURES
}

\author{
BRIAN JEFFERIES
}

\begin{abstract}
A theorem is given on the interchange of integrals for the product of a Radon polymeasure and a measure. Examples show that if the conditions of the theorem are not satisfied, then the conclusions may not hold.
\end{abstract}

The basic content of Fubini's theorem for the product of two $\sigma$-finite measure spaces, is that the integral of a function with respect to the product measure, when it exists, is equal to each of the iterated integrals with respect to the component measures. Standard examples show that the assumption of $\sigma$-finiteness cannot be omitted, and the iterated integrals of a function $f$ may themselves exist, without being equal to each other $[1$, p. 392].

The interchange of integrals with respect to the product of an operator-valued measure and a finite measure has proved to be of importance to the solution of operator equations arising from the perturbations of semigroups [5]. Extensions have also been developed for the cases where the operator-valued set function associated with the unperturbed semigroup is not $\sigma$-additive $[4,6]$.

The motivation for the present note arises from these applications, which are necessarily more involved than the cases considered here, because they deal with set functions with infinitely many variables. Nevertheless, similar features arise in the present context.

Given a separately $\sigma$-additive set function $m$ in finitely many variables, and a finite measure $\mu$, if a function $f$ is $m \times \mu$-integrable, then the iterated integral with the $\mu$-integral taken first and then the $m$-integral is equal to the integral with respect to $m \times \mu$. However, in reversing this order of integration, the $m$-integral, taken first, may not exist, and even if it does, the iterated integral may not be equal to the $m \times \mu$-integral.

The last case may be viewed as truly 'pathological', as for example, when the variation $|m|$ of $m$ fails to be $\sigma$-finite, or curiously enough, when $m$ has more than two variables.

Integration with respect to separately $\sigma$-additive set functions, or polymeasures, as introduced in [2], is reviewed in Section 1 . The main result, Theorem 4, on the

Received 11 November 1987

Research supported by a Queen Elizabeth II Fellowship

Copyright Clearance Centre, Inc. Serial-fee code: 0004-9729/88 \$A2.00+0.00. 
interchange of integrals with respect to the product of a Radon polymeasure and a finite measure is given in Section 2. Examples there show that even for the product of two measures, considered as a bimeasure, one iterated integral may fail to exist although the product integral does exist; it turns out that when both iterated integrals exist, they must be equal.

An example is also given of a function integrable with respect to the product of a polymeasure $m$ in three variables, and a finite measure for which both iterated integrals exist, but differ. As required by Theorem 4, the variation of $m$ is not $\sigma$-finite, and $m$ is not compact in the sense described in Section 2.

\section{Radon Polymeasures}

The notion of a Radon polymeasure was introduced in [2], and the integral with respect to such an additive set function was defined. A typical example arises in the following manner. Let $\triangle$ be the self-adjoint Laplacian operator on $L^{2}\left(\mathbf{R}^{d}\right)$, and let $U=e^{i \Delta}$ be the unitary operator defined by the operational calculus for self-adjoint operators. Then for a function $\phi \in \mathbf{L}^{2}\left(\mathbf{P}^{d}\right) \backslash \mathbf{L}^{1}\left(\mathbb{R}^{d}\right)$, the set function $A \times B \rightarrow\left(U\left(\phi \chi_{A}\right), \phi \chi_{B}\right)$, for all Borel subsets $A, B$ of $\mathbf{R}^{d}$ is separately $\sigma$-additive, but it does not extend to a $\sigma$-additive measure on the product space $\mathbb{R}^{d} \times \mathrm{P}^{d}$. The set function so defined is a prototype of those arising from Feymman integrals.

Let $X_{1}, X_{2}, \ldots, X_{n}$ be Hausdorff topological spaces. The family of products of sets in the Borel $\sigma$-algebras of the spaces is denoted by $\mathcal{B}\left(X_{1}\right) \times \mathcal{B}\left(X_{2}\right) \times \ldots \times \mathcal{B}\left(X_{n}\right)$; it is a semi-algebra of subsets of $X_{1} \times X_{2} \times \ldots \times X_{n}$. An additive set function $m: \mathcal{B}\left(X_{1}\right) \times$ $\mathcal{B}\left(X_{2}\right) \times \ldots \times \mathcal{B}\left(X_{2}\right) \rightarrow \mathbb{C}$ is called a Radon polymeasure if it is a (signed) Radon measure in each component, and its variation is the restriction to $\mathcal{B}\left(X_{1}\right) \times \mathcal{B}\left(X_{2}\right) \times \ldots \times \mathcal{B}\left(X_{2}\right)$ of a (possibly infinite) Radon measure on $X_{1} \times X_{2} \times \ldots \times X_{n},($ see [7]).

For a Hausdorff topological space $T$, denote by $\mathcal{K}_{T}$ the family of all compact subsets of $T$. The variation of $m$ on sets $K_{1} \times K_{2} \times \ldots \times K_{n}, K_{i} \in \mathcal{K}_{X_{i}}, i=1,2, \ldots, n$ is denoted by $|m|_{K_{1} \times K_{2} \times \ldots \times K_{n}}$. The $\sigma$-additive extension of $|m| K_{1} \times K_{2} \times \ldots \times K_{n}$ to $\mathcal{B}\left(K_{1} \times K_{2} \times \ldots \times K_{n}\right)$ is denoted by the same symbol. The restriction of $m$ to $\mathcal{B}\left(K_{1}\right) \times \mathcal{B}\left(K_{2}\right) \times \ldots \times \mathcal{B}\left(K_{n}\right)$ also has a $\sigma$-additive extension $m_{K_{1} \times K_{2} \times \ldots \times K_{n}}$ to $\mathcal{B}\left(K_{1} \times K_{2} \times \ldots \times K_{n}\right)$.

A function $f: X_{1} \times X_{2} \times \ldots \times X_{n} \rightarrow C$ is said to be $m$-integrable if for every $K_{i} \in \mathcal{K}_{X_{i}}, i=1,2, \ldots, n$, its restriction $f_{K_{1} \times K_{2} \times \ldots \times K_{n}}$ to $K_{1} \times K_{2} \times \ldots \times K_{n}$ is $|m|_{K_{1} \times K_{2} \times \ldots \times K_{n}}$-integrable, and there exists a Radon polymeasure $f m: \mathcal{B}\left(X_{1}\right) \times$ $\mathcal{B}\left(X_{2}\right) \times \ldots \times \mathcal{B}\left(X_{n}\right) \rightarrow \mathbb{C}$ such that

$$
f m\left(K_{1} \times \ldots \times K_{n}\right)=f_{K_{1} \times \ldots \times K_{n}} m_{K_{1} \times \ldots \times K_{n}}\left(K_{1} \times \ldots \times K_{n}\right), K_{i} \in \mathcal{K}_{X_{i}}, i=1, \ldots, n
$$

In a similar fashion, the integral with respect to the product 
$m \times \mu: \mathcal{B}\left(X_{1}\right) \times \mathcal{B}\left(X_{2}\right) \times \ldots \times \mathcal{B}\left(X_{n}\right) \times \mathcal{T} \rightarrow \mathbb{C}$ of a measure $\mu: \mathcal{T} \rightarrow[0, \infty[$ and a Radon polymeasure $m: \mathcal{B}\left(X_{1}\right) \times \mathcal{B}\left(X_{2}\right) \times \ldots \times \mathcal{B}\left(X_{n}\right) \rightarrow \mathcal{C}$ can be defined.

Let $\mathcal{T}$ be a $\sigma$-algebra of subsets of a set $\Sigma$, and suppose that $\mu: \mathcal{T} \rightarrow[0, \infty[$ is a finite measure on $\mathcal{T}$. Set $\Omega=X_{1} \times X_{2} \times \ldots \times X_{n}$. A function $f: \Omega \times \Sigma \rightarrow \mathbb{C}$ is said to be $m \times \mu$-integrable if for every $K_{i} \in \mathcal{K}_{X_{i}}, i=1,2, \ldots, n$, the restriction $f_{K_{1} \times \ldots \times K_{n}}$ of $f$ to $K_{1} \times \ldots \times K_{n} \times \Sigma$ is $|m|_{K_{1} \times K_{2} \times \ldots \times K_{n}} \times \mu$-integrable, and there exists a set function $f m \times \mu: \mathcal{B}\left(X_{1}\right) \times \mathcal{B}\left(X_{2}\right) \times \ldots \times \mathcal{B}\left(X_{n}\right) \times \mathcal{T} \rightarrow \mathbb{C}$ such that for every $T \in \mathcal{T}$, the set function

$$
A_{1} \times A_{2} \times \ldots \times A_{n} \rightarrow f m \times \mu\left(A_{1} \times A_{2} \times \ldots \times A_{n} \times T\right), A_{i} \in \mathcal{B}\left(X_{i}\right), i=1,2, \ldots, n
$$

is a Radon polymeasure, $T \rightarrow f m \times \mu\left(A_{1} \times A_{2} \times \ldots \times A_{n} \times T\right), T \in \mathcal{T}$ is $\sigma$-additive for every $A_{i} \in \mathcal{B}\left(X_{i}\right), i=1,2, \ldots, n$, and

$$
f m \times \mu\left(K_{1} \times \ldots \times K_{n} \times T\right)=f_{K_{1} \times \ldots \times K_{n}} m_{K_{1} \times \ldots \times K_{2}} \otimes \mu\left(K_{1} \times \ldots \times K_{n} \times T\right)
$$

for every $K_{i} \in \mathcal{K}_{X_{i}}, i=1,2, \ldots, n, T \in \mathcal{T}$. So defined, the indefinite integral of the function $f$ with respect to $m \times \mu$ is unique.

\section{Product integration}

The first observation to make about integration with respect to the product of a Radon polymeasure $m$ and a finite measure $\mu$, is that the product integral of a function is equal to the iterated integral, as long as we integrate with respect to the measure first.

Proposition 1. Let $m: \mathcal{B}\left(X_{1}\right) \times \mathcal{B}\left(X_{2}\right) \times \ldots \times \mathcal{B}\left(X_{n}\right) \rightarrow \mathbb{C}$ be a Radon polymeasure, and suppose that $\mu: \mathcal{T} \rightarrow[0, \infty \mid$ is a finite measure on the $\sigma$-algebra $\mathcal{T}$ of subsets of the set $\Sigma$. Let $f: X_{1} \times X_{2} \times \ldots \times X_{n} \times \Sigma \rightarrow \mathbb{C}$ be an $m \times \mu$-integrable function. Set $\Omega=X_{1} \times X_{2} \times \ldots \times X_{n}$.

Then there exists a set $\Omega_{0} \subseteq \Omega$ such that $|m|\left(\Omega \backslash \Omega_{0}\right)=0$, for every $\omega \in$ $\Omega_{0}$, the function $\sigma \rightarrow f(\omega, \sigma), \sigma \in \Sigma$, is $\mu$-integrable, and the function $\omega \rightarrow$ $\int_{\Sigma} f(\omega, \sigma) d \mu(\sigma), \omega \in \Omega_{0}$ is m-integrable. Furthermore,

$$
f m \times \mu(A \times B)=\int_{A} \int_{B} f(\omega, \sigma) d \mu(\sigma) d m(\omega), A \in \mathcal{B}\left(X_{1}\right) \times \ldots \times \mathcal{B}\left(X_{n}\right), B \in \mathcal{T}
$$

Proof: Write $f=f^{+}-f^{-}$. for functions $f^{+}, f^{-} \geqslant 0$. For every compact set $K \subset \Omega$, the function $f \chi_{K}$ is $|m|_{K} \otimes \mu$-integrable. Since $|m|$ is a Radon measure, $f$ is $|m| \otimes \mu$-measurable, so there exist increasing sequences of $|m| \otimes \mu$-simple functions $s_{k}^{+}, s_{k}^{-}, k=1,2, \ldots$ such that $s_{k}^{+} \rightarrow f^{+}, s_{k}^{-} \rightarrow f^{-}$as $k \rightarrow \infty$. 
The complement $\Omega \backslash \Omega_{0}$ of the set $\Omega_{0}$ of points $\omega \in \Omega$ for which the sequences $\int_{\Sigma} s_{k}^{+}(\omega, \sigma) d \mu(\sigma), \int_{\Sigma} s_{k}^{+}(\omega, \sigma) d \mu(\sigma), k=1,2, \ldots$ converge is $|m|$-null, because $|m|\left(\left(\Omega \backslash \Omega_{0}\right) \cap K\right)=0$ for every compact set $K \subset \Omega$.

The conclusion now follows, because for every $K_{i} \in \mathcal{K}_{X_{i}}, i=1,2, \ldots, n, B \in \mathcal{T}$, the equality

$$
f m \times \mu\left(K_{1} \times \ldots \times K_{n} \times B\right)=\int_{K_{1} \times \ldots \times K_{n}} \cdots \int_{B} f(\omega, \sigma) d \mu(\sigma) d m(\omega)
$$

holds by virtue of the usual version of Fubini's theorem, and $f m \times \mu$ is a Radon polymeasure in the first $n$ variables.

The following example shows that even for the product of two measures, a function $f$ may be integrable in the sense of bimeasures without one of the iterated integrals existing.

Example 1. Let $f:] 0,1] \times[0,1] \rightarrow \mathbb{R}$ be the function defined by $f(x, t)=$ $2^{k+1} \sin (k t) / k$ for $t \in[0,1]$ and $\left.\left.x \in\right] 2^{-k-1}, 2^{-k}\right], k=1,2, \ldots$, and 0 elsewhere.

The sequence $\{\sin (k \cdot) / k\}_{k=1}^{\infty}$ is summable in $\mathbf{L}^{1}([0,1])$, so it is easy to check that $m(A \times B)=\iint_{A} f(x, t) d t d x, A, B \in \mathcal{B}([0,1])$ is separately $\sigma$-additive on $\left.] 0,1\right] \times$ $[0,1]$, and it is Radon bimeasure on the space $] 0,1] \times[0,1]$.

Let $\lambda$ be the Lebesgue measure on $[0,1]$. The function $f$ is $\lambda \times \lambda$-integrable on $] 0,1] \times[0,1]$, in the sense of bimeasures, because the sequence $\{\sin (k \cdot) / k\}_{k=1}^{\infty}$ is summable in $\mathbf{L}^{1}([0,1])$.

Now for every $t \in[0,1], \int_{0}^{1}|f(x, t)| d x=\sum_{k=1}^{\infty} \frac{|\sin (k t)|}{k}$, which diverges almost everywhere on $[0,1]$, so for almost every $t \in[0,1]$, the function $x \rightarrow f(x, t), x \in] 0,1]$ is not $\lambda$-integrable.

The next proposition ensures that when the iterated integrals do exist, they must be equal for the case of the product of two measures.

Let $\Omega$ be a Hausdorff topological space, let $\lambda$ be finite Radon measures on $\Omega$, and let $\mu: \mathcal{T} \rightarrow[0, \infty[$ be a finite measure on the $\sigma$-algebra $\mathcal{T}$ of subsets of $\Sigma$. Suppose that the function $f: \Omega \times \Sigma \rightarrow \mathbf{R}$ is $\lambda \times \mu$-integrable in the sense of Radon bimeasures, described in Section 1.

Proposition 2. If for $\mu$-almost all $\sigma \in \Sigma$ the function $f(\cdot, \sigma)$ is $\lambda$-integrable, then $\int_{\Sigma}\left|\int_{A} f(\omega, \sigma) d \lambda(\omega)\right| d \mu(\sigma)<\infty$, and

$$
f \lambda \times \mu(A \times B)=\int_{B} \int_{A} f(\omega, \sigma) d \lambda(\omega) d \mu(\sigma), A \in \mathcal{B}(\Omega), B \in \mathcal{B}(\Sigma) .
$$


Proof: Let $\Sigma_{0}$ be the set of all $\sigma \in \Sigma$ for which the function $f(\cdot, \sigma)$ is $\lambda$ integrable. The function $f$ may be assumed to be $\mathcal{B}(\Omega) \otimes \mathcal{T}$-measurable after modification on a $|\lambda| \otimes \mu-$ null set, if necessary. By the assumption, $\mu\left(\Sigma \backslash \Sigma_{0}\right)=0$.

Let $A \in \mathcal{B}(\Omega), B \in \mathcal{T}$. For every $k=1,2, \ldots$, the set $K_{k} \in \mathcal{T}$ is defined by $K_{k}=\left\{\sigma \in \Sigma: \int_{\Omega}|f(\omega, \sigma)| d \lambda(\omega) \leqslant k\right\}$. Then $K_{k} \subseteq K_{k+1}, k=1,2, \ldots$, and $\bigcup_{k=1}^{\infty} K_{k}=\Sigma_{0}$, and by Fubini's Theorem,

$$
f \lambda \times \mu\left(A \times B \cap K_{k}\right)=\int_{B \cap K_{k}} \int_{A} f(\omega, \sigma) d \lambda(\omega) d \mu(\sigma) .
$$

The convergence as $k \rightarrow \infty$ is uniform for $B \in \mathcal{B}(\Sigma)$ because $f \lambda \times \mu$ is $\sigma$-additive in the second variable. For every compact set $C \subset \Omega$, and every set $B \in \mathcal{B}(\Sigma)$, $f \lambda \times \mu\left(C \times B \cap\left(\Sigma \backslash \Sigma_{0}\right)\right)=0$ by Fubini's theorem, so the result follows.

The same argument can be made to work for the case where the Radon measure $\lambda$ is replaced by a Radon polymeasure $m$, provided some additional assumptions are made. First it is necessary to consider the tensor product of spaces of measures.

Let $E, F$ be two Banach spaces. The injective tensor product norm $\|x \otimes y\|_{\varepsilon}$ of the tensor product $x \otimes y$ of $x \in E, y \in F$ is defined by

$$
\|x \otimes y\|_{\epsilon}-\sup \left\{\left|\langle x, \xi\rangle\left\|\langle y, \zeta\rangle \mid: \xi \in E^{\prime}, \zeta \in F^{\prime},\right\| \xi\|\leqslant 1,\| \zeta \| \leqslant 1\right\} .\right.
$$

The completion of $E \otimes F$ in the norm $\|\cdot\|_{e}$ is denoted by $E \otimes_{\epsilon} F$.

A Radon polymeasure $m: \mathcal{B}\left(X_{1}\right) \times \mathcal{B}\left(X_{2}\right) \times \ldots \times \mathcal{B}\left(X_{n}\right) \rightarrow \mathbb{C}$ is said to be compact if $m$ belongs to the tensor product $\mathbf{M}\left(\mathcal{B}\left(X_{1}\right)\right) \otimes_{\varepsilon} \ldots \otimes_{\varepsilon} \mathbf{M}\left(\mathcal{B}\left(X_{n}\right)\right)$ of the spaces $\mathbf{M}\left(\mathcal{B}\left(X_{1}\right)\right), \ldots, \mathbf{M}\left(\mathcal{B}\left(X_{n}\right)\right)$ of measures.

The terminology is derived from the case where $n=2$, when $m$ is compact precisely when the associated vector measure with values in a space of measures has compact range. A Radon polymeasure $m$ is compact when it can be approximated uniformly on the semi-algebra $\mathcal{B}\left(X_{1}\right) \times \mathcal{B}\left(X_{2}\right) \times \ldots \times \mathcal{B}\left(X_{n}\right)$ by finite linear combinations of product measures.

The compactness of a polymeasure has a bearing on the nature of its $\sigma$-additivity; the relationship is explored in [3] from which the following result is taken. Set $\Omega=$ $X_{1} \times X_{2} \times \ldots \times X_{n}$.

Proposition 3. Let $m: \mathcal{B}\left(X_{1}\right) \times \mathcal{B}\left(X_{2}\right) \times \ldots \times \mathcal{B}\left(X_{n}\right) \rightarrow \mathbb{C}$ be a compact Radon polymeasure.

The $\sigma$-additivity of $m$ in each variable is uniform with respect to the other variables: if $A_{k} \in \mathcal{B}\left(X_{1}\right), A_{k} \downarrow \emptyset$ then $m\left(A_{k} \cap B_{1} \times B_{2} \times \ldots \times B_{n}\right) \rightarrow 0$ in $C$, uniformly for all $B_{j} \in \mathcal{B}\left(X_{j}\right), j=1,2, \ldots, n$. 
Furthermore, $m$ is inner regular in each variable, uniformly with respect to the other variables; for each $A \in \mathcal{B}\left(X_{1}\right)$, and each $\varepsilon>0$, there exists a compact set $K \subset A$ such that $\left|m\left((A \backslash K) \cap B_{1} \times B_{2} \times \ldots \times B_{n}\right)\right|<\varepsilon$ for all $B_{j} \in \mathcal{B}\left(X_{j}\right), j=1,2, \ldots, n$.

Theorem 4. Suppose that $m$ is a Radon polymeasure on $\Omega$, and that the function $f: \Omega \times \Sigma \rightarrow \mathbf{R}$ is $m \times \mu$-integrable in the sense of polymeasures, and for a set $\Sigma_{0} \subseteq \Sigma$, such that $\mu\left(\Sigma \backslash \Sigma_{0}\right)=0$, the function $f(\cdot, \sigma)$ is m-integrable for every $\sigma \in \Sigma_{0}$. Suppose that one of the following conditions hold:

(i) the variation $|m|$ of $m$ is $\sigma$-finite.

(ii) $m$ is a bimeasure;

(iii) $m$ is compact, and for each $\sigma \in \Sigma_{0}$, the Radon polymeasure $f(\cdot, \sigma) m$ is compact.

Then $\int_{\Sigma}\left|\int_{A} f(\omega, \sigma) d m(\omega)\right| d \mu(\sigma)<\infty$, and

$$
f m \times \mu(A \times B)=\int_{B} \int_{A} f(\omega, \sigma) d m(\omega) d \mu(\sigma), A \in \mathcal{B}(\Omega), B \in \mathcal{B}(\Sigma) .
$$

Proof: If condition (i) holds, then there exists an increasing sequence of compact subsets $K_{i}, i=1,2, \ldots$ of $\Omega$ such that $|m|\left(\Omega \backslash \bigcup_{i \in \mathbb{N}} K_{i}\right)=0$. Let $\Pi_{j}: \Omega \rightarrow \Omega_{j}, j=$ $1, \ldots, n$ be the natural projections of $\Omega$ onto its components, and set

$$
C_{1 j_{1}}=\Pi_{1}\left(K_{j_{1}}\right), C_{2 j_{2}}=\Pi_{2}\left(K_{j_{2}}\right), \ldots, C_{n j_{n}}=\Pi_{n}\left(K_{j_{n}}\right), j_{1}, \ldots, j_{n}=1,2, \ldots
$$

Then

$$
\begin{array}{r}
m\left(\left(X_{1} \backslash \bigcup_{j_{1} \in N} C_{1 j_{1}}\right) \bigcap A_{1} \times A_{2} \times \ldots \times A_{n}\right)=0 \\
\vdots \\
m\left(A_{1} \times A_{2} \times \ldots \times\left(X_{n} \backslash \bigcup_{j_{n} \in \mathbb{N}} C_{n j_{n}}\right) \bigcap A_{n}\right)=0
\end{array}
$$

for every $A_{1} \in \mathcal{B}\left(X_{1}\right), \ldots, A_{n} \in \mathcal{B}\left(X_{n}\right)$, because the equalities hold whenever $A_{1}, \ldots, A_{n}$ are compact subsets of $X_{1}, \ldots, X_{n}$. It follows that for each $\sigma \in \Sigma_{0}$.

$$
\begin{gathered}
f(\cdot, \sigma) m\left(\left(X_{1} \backslash \bigcup_{j_{1} \in \mathbb{N}} C_{1 j_{1}}\right) \bigcap A_{1} \times A_{2} \times \ldots \times A_{n}\right)=0 \\
\vdots \\
f(\cdot, \sigma) m\left(A_{1} \times A_{2} \times \ldots \times\left(X_{n} \backslash \bigcup_{j_{n} \in \mathbb{N}} C_{n j_{n}}\right) \bigcap A_{n}\right)=0 .
\end{gathered}
$$


Let $A \in \mathcal{B}\left(X_{1}\right) \times \ldots \times \mathcal{B}\left(X_{n}\right)$. Set

$$
K_{k}=\left\{\sigma \in \Sigma_{0}: \sup _{j_{1} \in N, \ldots, j_{n} \in N}\left|\int_{A \cap C_{1 j_{1}} \times \ldots \times C_{n j_{n}}} f(\omega, \sigma) d m(\omega)\right| \leqslant k\right\} .
$$

The sets $K_{k}, k=1,2, \ldots$ are $\mu$-measurable, and $\mu\left(\Sigma \backslash \bigcup_{k \in \mathbb{N}} K_{k}\right)=0$. Then by Fubini's theorem,

$$
\begin{aligned}
f m \times \mu\left(\left(A \bigcap C_{1 j_{1}} \times \ldots \times C_{n j_{n}}\right) \times B \bigcap K_{k}\right) & \\
= & \int_{B \cap K_{k}} \int_{A \cap C_{1 j_{1}} \times \ldots \times C_{n j_{n}}} f(\omega, \sigma) d m(\omega) d \mu(\sigma), \\
f m \times \mu(A \times B & \left.\bigcap K_{k}\right) \\
& =\int_{B \cap K_{k}} \int_{A} f(\omega, \sigma) d m(\omega) d \mu(\sigma)
\end{aligned}
$$

because we can take the limit as $j_{1}, \ldots, j_{n} \rightarrow \infty$ respectively, and appeal to dominated convergence, and the assumption that for each $\sigma \in \Sigma_{0}, f(\cdot, \sigma) m$ is a polymeasure.

The convergence as $k \rightarrow \infty$ is uniform for $B \in \mathcal{B}(\Sigma)$ because $f m \times \mu$ is $\sigma$-additive in the second variable, so the result follows.

If $m$ is a bimeasure, or condition (iii) holds, then there exist increasing families $C_{1 j_{1}}, C_{2 j_{2}}, \ldots, C_{n j_{n}}, j_{1}, \ldots, j_{n}=1,2, \ldots$ of compacts sets such that

$$
\begin{array}{r}
\lim _{j_{1} \rightarrow \infty} \sup \{\mid m \\
\left(\left(X_{1} \backslash C_{1 j_{1}}\right) \bigcap A_{1} \times A_{2} \times \ldots \times A_{n}\right) \mid \\
\left.: A_{1} \in \mathcal{B}\left(X_{1}\right), \ldots, A_{n} \in \mathcal{B}\left(X_{n}\right)\right\}=0 \\
\vdots \\
\lim _{j_{n} \rightarrow \infty} \sup \left\{\left|m\left(A_{1} \times A_{2} \times \ldots \times\left(X_{n} \backslash C_{n j_{n}}\right) \bigcap A_{n}\right)\right|\right. \\
\left.: A_{1} \in \mathcal{B}\left(X_{1}\right), \ldots, A_{n} \in \mathcal{B}\left(X_{n}\right)\right\}=0 .
\end{array}
$$

For each $\sigma \in \Sigma_{0}$, the Radon polymeasure $n_{\sigma}=f(\cdot, \sigma) m$ is compact, or it is a bimeasure, so in either case, it lies in the closure of the set

$$
\left\{s m: s: \Omega \rightarrow \mathrm{R} \text { is } \mathcal{B}\left(X_{1}\right) \times \ldots \times \mathcal{B}\left(X_{n}\right)-\text { simple }\right\}
$$

in the topology of uniform convergence on the semi-algebra $\mathcal{B}\left(X_{1}\right) \times \ldots \times \mathcal{B}\left(X_{n}\right)$. This follows by approximating $n_{\sigma}$ by its restrictions to compact subsets of $\Omega$, by virtue of Proposition 3. 
It follows that for each $\sigma \in \Sigma_{0}$.

$$
\begin{array}{r}
\lim _{j_{1} \rightarrow \infty} \sup \left\{\left|n_{\sigma}\left(\left(X_{1} \backslash C_{1 j_{1}}\right) \bigcap A_{1} \times A_{2} \times \ldots \times A_{n}\right)\right|\right. \\
\left.: A_{1} \in \mathcal{B}\left(X_{1}\right), \ldots, A_{n} \in \mathcal{B}\left(X_{n}\right)\right\}=0 \\
\vdots \\
\lim _{j_{n} \rightarrow \infty} \sup \left\{\left|n_{\sigma}\left(A_{1} \times A_{2} \times \ldots \times\left(X_{n} \backslash C_{n j_{n}}\right) \bigcap A_{n}\right)\right|\right. \\
\left.: A_{1} \in \mathcal{B}\left(X_{1}\right), \ldots, A_{n} \in \mathcal{B}\left(X_{n}\right)\right\}=0 .
\end{array}
$$

Let $A \in \mathcal{B}\left(X_{1}\right) \times \ldots \times \mathcal{B}\left(X_{n}\right)$. For each $k=1,2, \ldots$, set

$$
K_{k}=\left\{\sigma \in=\Sigma_{0}: \sup _{j_{1} \in N_{1}, \ldots, j_{n} \in N}\left|\int_{A \cap C_{1 j_{1}} \times \ldots \times C_{n j_{n}}} f(\omega, \sigma) d m(\omega)\right| \leqslant k\right\} .
$$

Now the same argument as in part (i) applies.

The following example shows that the conclusion of the theorem may not hold if one of the above conditions is not satisfied.

Example 2. Let $(\Gamma, S, \mu)$ be a compact probability measure space, and let $\left\langle f_{\sigma}\right\rangle_{\sigma \in[0,1]}$ be an orthonormal basis of $L^{2}(\mu)$ such that $\left|f_{\sigma}\right|=1$ for each $\sigma \in[0,1]$; the functions $f_{\sigma}, \sigma \in[0,1]$ are complex valued. Let $\lambda$ be the Lebesgue measure on $[0,1]$.

For example $\Gamma$ may be the product of $2^{\aleph_{0}}$-copies of the torus, with $\mu$ the normalised Haar measure on $\Gamma$. The basis $\left\langle f_{\sigma}\right\rangle_{\sigma \in[0,1]}$ could then be cliosen to be the collection of finite products of characters on $\Gamma$, arranged suitably.

For every $A \subseteq[0,1], B, C \in S$ set

$$
m(A \times B \times C)=\sum_{\sigma \in A}\left(f_{\sigma}, \chi_{B}\right)\left(f_{\sigma}, \chi_{C}\right) .
$$

Clearly $m$ is a trimeasure on $[0,1] \times \Gamma \times \Gamma$ where $[0,1]$ is endowed with the discrete topology. Let $\nu$ be the counting measure on $[0,1]$. Considering $f_{\sigma}(x) f_{\sigma}(y), \sigma \in$ $[0,1], x, y \in \Gamma$ as a function $f$ of three variables on $[0,1] \times \Gamma \times \Gamma$, for all compact sets II $\subset\{0,1], L, M \subset \Gamma$,

$$
m(K \times L \times M)=f|\nu \otimes \mu \otimes \mu|(K \times L \times M) .
$$

Let $D$ be the set $\{(\sigma, \sigma, x, y): \sigma \in[0,1], x, y \in \Gamma\}$. Define the function $\Psi:[0,1] \times$ $[0,1] \times \Gamma \times \Gamma \rightarrow R$ by

$$
\Psi(\sigma, \tau, x, y)=\chi_{D}(\sigma, \tau, x, y) / f(\tau, x, y)
$$


for every $\sigma, \tau \in[0,1], x, y \in \Gamma$.

Then for every $\tau \in[0,1], x, y \in \Gamma$, the function $\sigma \rightarrow \Psi(\sigma, \tau, x, y), \sigma \in[0,1]$ is $\lambda$-integrable and $\Psi(\cdot, \tau, x, y) \lambda=0$. Therefore, the function $\Psi$ is $m \times \lambda$-integrable, and $\Psi m \times \lambda=0$.

Moreover, for ever $\sigma \in[0,1]$,

$$
\begin{aligned}
\int_{[0,1] \times \Gamma \times \Gamma} \Psi(\sigma, \tau, x, y) d m(\tau, x, y) & =\int_{[0,1] \times \Gamma \times \Gamma} \chi_{D}(\sigma, \tau, x, y) d \nu \otimes \mu \otimes \mu(\tau, x, y) \\
& =1,
\end{aligned}
$$

so

$$
\int_{0}^{1}\left[\int_{[0,1] \times \Gamma \times \Gamma} \Psi(\sigma, \tau, x, y) d m(\tau, x, y)\right] d \lambda(\sigma)=1 .
$$

Although the iterated integrals both exist, they are not equal.

\section{REFERENCES}

[1] E. Hewitt and K. Stromberg, Real and Abstract Analysis (Springer-Verlag, New York, Heidelberg, Berlin, 1965).

[2] B. Jefferies, 'Radon polymeasures', Bull. Austral. Math. Soc. 32 (1985), 207-215.

[3] B. Jefferies (to appear), Summability in spaces of set functions to appear. Proc. Ramanujan Birth Centenary Year Symp. on Anal., Pune.

[4] B. Jefferies, 'Integration with respect to the product of a measure and a closable set function'. (submitted) .

[5] I. Kluvánek, 'Operator valued measures and perturbations of semigroups', Arch. Rat. Mech. Anal. 81 (1983), 161-180.

[6] I. Kluvánek, 'Integration and the Feynman-Kac formula', Studia Math. 86 (1987), 35-57.

[7] L. Scliwartz, Radon Measures on Arbitrary Topological Spaces (Oxford University Press, 1973).

Department of Mathematics

The University of Wollongong

P.O. Box 1144

Wollongong N.S.W. 2500

Australia 